\title{
Second-Order Moment Convergence Rates for Spectral Statistics of Random Matrices
}

\author{
Junshan Xie \\ College of Mathematics and Information, Henan University, Kaifeng 475000, China \\ Correspondence should be addressed to Junshan Xie; junshan@henu.edu.cn
}

Received 13 June 2013; Accepted 21 October 2013

Academic Editor: Henrik Kalisch

Copyright (C) 2013 Junshan Xie. This is an open access article distributed under the Creative Commons Attribution License, which permits unrestricted use, distribution, and reproduction in any medium, provided the original work is properly cited.

This paper considers the precise asymptotics of the spectral statistics of random matrices. Following the ideas of Gut and Spătaru (2000) and Liu and Lin (2006) on the precise asymptotics of i.i.d. random variables in the context of the complete convergence and the second-order moment convergence, respectively, we will establish the precise second-order moment convergence rates of a type of series constructed by the spectral statistics of Wigner matrices or sample covariance matrices.

\section{Introduction and Main Results}

This paper is concerned with the precise asymptotic behaviors of the spectral (eigenvalue) statistics of random matrices; two types of classical random matrices including Wigner matrices and sample covariance matrices will be considered. An $n \times n$ Wigner matrix is defined to be a random Hermitian matrix $X_{n}=\left(X_{i j}\right)_{1 \leq i, j \leq n}$, in which the real and imaginary parts of $X_{i j}$ for $i<j$ are i.i.d. random variables with mean 0 and variance $1 / 2$ (in that case, $\mathbb{E} X_{i j}^{2}=0$ ), and $X_{i i}, i=1,2, \ldots, n$, are i.i.d. random variables with mean 0 and variance 1 . Denote by $\lambda_{1} \leq \cdots \leq \lambda_{n}$ the real eigenvalues of the normalized Wigner matrix $M_{n}=(1 / \sqrt{n}) X_{n}$. The classical Wigner theorem states that the empirical distribution $F^{M_{n}}(x)=(1 / n) \sum_{i=1}^{n} 1_{\left\{\lambda_{i} \leq x\right\}}$ converges almost surely to the semicircle law with the density $\mu_{s c}(x)=(1 / 2 \pi) \sqrt{4-x^{2}} 1_{[-2,2]}(x)$. Consequently, for any bounded continuous function $f$, the spectral statistics satisfy that

$$
\begin{aligned}
\lim _{n \rightarrow \infty} \frac{1}{n} \sum_{i=1}^{n} f\left(\lambda_{i}\right) & =\lim _{n \rightarrow \infty} \int f(x) d F^{M_{n}}(x) \\
& =\int f(x) \mu_{s c}(x) d x \text { a.s. }
\end{aligned}
$$

The result can be viewed as an analog of the law of large number for independent random variables. As for the fluctuation of the spectral statistics, a remarkable work due to Bai et al. [1] states the following.

Lemma 1. Assume the entries of a Wigner matrix $X_{n}$ satisfy that $E\left|X_{i j}\right|^{4}=2$ and $E\left|X_{i j}\right|^{6}<\infty$ for all $1 \leq i, j \leq n$. Let $\Pi$ be an open interval including the interval $[-2,2]$ and $C^{4}(\Pi)$ is the space of forth-order continuous differentiable functions on П. Denote

$$
G_{n}(f):=n\left\{\int f(x) d F^{M_{n}}(x)-\int f(x) \mu_{s c}(x) d x\right\} .
$$

Then the empirical process $\left\{G_{n}(f): f \in C^{4}(\Pi)\right\}$ converges weakly in finite dimension to a Gaussian process $\{G(f): f \in$ $\left.C^{4}(\Pi)\right\}$ with mean zero and the covariance function $\operatorname{Cov}(f, g)$ given by

$$
\begin{aligned}
& \operatorname{Cov}(f, g) \\
& \begin{aligned}
=\frac{1}{4 \pi^{2}} \iint_{-2}^{2} f^{\prime}(t) g^{\prime}(s) \\
\quad \times \log \left(\frac{4-t s+\sqrt{\left(4-t^{2}\right)\left(4-s^{2}\right)}}{4-t s-\sqrt{\left(4-t^{2}\right)\left(4-s^{2}\right)}}\right) d t d s .
\end{aligned}
\end{aligned}
$$

In addition, Guionnet and Zeitouni [2] give a concentration inequality on the empirical spectral measure of $F^{M_{n}}(x)$ near the semicircle law. 
Lemma 2. Assume the law of the entries $\left\{\operatorname{Re}\left(X_{i j}\right), \operatorname{Im}\left(X_{i j}\right)\right.$, $\left.X_{i i}\right\}$ satisfies the logarithmic Sobolev inequality (LSI) with constant $c_{0}>0$. Let $f$ be a Lipschitz function and denote

$$
\eta_{n}(f)=n^{-1} G_{n}(f)=\int f(x) d F^{M_{n}}(x)-\int f(x) \mu_{s c}(x) d x .
$$

Then for any $\delta>\delta_{n}(f)=\mid \mathbb{E} \int f(x) d F^{M_{n}}(x)-$ $\int f(x) \mu_{s c}(x) d x \mid$, there exists $c>0$, such that

$$
P\left(\left|\eta_{n}(f)\right| \geq \delta\right) \leq 2 e^{-c n^{2}\left(\delta-\delta_{n}(f)\right)^{2}} .
$$

Based on the above existing results on the limiting spectral properties of random matrices, we will give another type of asymptotic spectral properties, that is, the precise asymptotics of the spectral statistics of random matrices. In particular, we will consider the precise second-order moment convergence rates of a type of series constructed by the spectral statistics of random matrices. Our first result can be listed as follows.

Theorem 3. Suppose that the law of the entries $\left\{\operatorname{Re}\left(X_{i j}\right)\right.$, $\left.\operatorname{Im}\left(X_{i j}\right), X_{i i}\right\}$ satisfies $L S I$ with constant $c_{0}>0$ and $E\left|X_{i j}\right|^{4}=2$ for all $1 \leq i, j \leq n$. Denote $\eta_{n}(f)$ as $(4)$, where $f \in C^{4}(\Pi)$ has a bounded first-order derivative. Then for any $s>1 / 2, \beta>2 s-1$, one has

$$
\begin{gathered}
\lim _{\epsilon \searrow 0} \epsilon^{(\beta+1) / s-2} \sum_{n=1}^{\infty} n^{\beta-2 s+2} \mathbb{E}\left|\eta_{n}(f)\right|^{2} I_{\left\{\left|\eta_{n}(f)\right| \geq \epsilon n^{s-1}\right\}} \\
=\frac{1}{\beta-2 s+1}[D(f)]^{(\beta+1) / 2 s} \mathbb{E}|\xi|^{(\beta+1) / s},
\end{gathered}
$$

where $\xi$ is a standard Gaussian random variable and

$$
\begin{aligned}
D(f)=\frac{1}{4 \pi^{2}} \iint_{-2}^{2} f^{\prime}(t) f^{\prime}(s) \\
\quad \times \log \left(\frac{4-t s+\sqrt{\left(4-t^{2}\right)\left(4-s^{2}\right)}}{4-t s-\sqrt{\left(4-t^{2}\right)\left(4-s^{2}\right)}}\right) d t d s .
\end{aligned}
$$

Now we turn to the sample covariance matrix, which is an important statistic in multivariate statistics analysis. Let $Y_{m n}=\left(Y_{i j}\right)_{1 \leq i \leq m, 1 \leq j \leq n}$ be an $m \times n$ random matrix whose entries are i.i.d. complex-valued random variables with mean 0 and variance 1 and $\operatorname{Re}\left(Y_{i j}\right)_{i<j}$ and $\operatorname{Im}\left(Y_{i j}\right)_{i<j}$ are independent random variables with mean 0 and variance $1 / 2$. Then $W_{m, n}=(1 / n) Y_{m n} Y_{m n}^{*}$ can be viewed as the sample covariance matrix of $n$ samples of $m$-dimensional random vectors. Let $\lambda_{1}^{\prime} \leq \cdots \leq \lambda_{m}^{\prime}$ be the eigenvalues of $W_{m, n}$, and define the empirical spectral distribution $F^{W_{m, n}}(x)=$ $(1 / m) \sum_{i=1}^{m} I_{\left\{\lambda_{i}^{\prime} \leq x\right\}}$. When $m / n \rightarrow \rho \in(0,1]$ as $n \rightarrow \infty$, the famous Marchenko-Pastur theorem reveals that almost surely $F^{W_{m, n}}(x)$ converges to the Marchenko-Pastur law with the density $\gamma_{\rho}(x)=(1 / 2 \pi x \rho) \sqrt{(b(\rho)-x)(x-a(\rho))} 1_{[a(\rho), b(\rho)]}(x)$, where $a(\rho)=(1-\sqrt{\rho})^{2}, b(\rho)=(1+\sqrt{\rho})^{2}$.
Denote

$$
\tau_{m, n}(f)=\int f(x) d F^{W_{m, n}}(x)-\int f(x) \gamma_{m / n}(x) d x,
$$

where $\gamma_{m / n}(x)$ is the Marchenko-Pastur law $\gamma_{\rho}(x)$ with the parameter $\rho=m / n$. Similar to the result on Wigner matrices, we can give the result on the complex sample covariance matrices.

Theorem 4. Assume the law of the entries $\left\{\operatorname{Re}\left(Y_{i j}\right), \operatorname{Im}\left(Y_{i j}\right)\right\}$ satisfies LSI with constant $c_{0}>0$ and $E\left|Y_{i j}\right|^{4}=2$ for all $1 \leq i \leq$ $m, 1 \leq j \leq n, m=[n \rho](0<\rho \leq 1)$. If one denotes $\Gamma$ to be an open interval including $[a(\rho), b(\rho)], f \in C^{4}(\Gamma)$ has a bounded first order derivative. Then for any $s>1 / 2, \beta>2 s-1$, one has

$$
\begin{gathered}
\lim _{\epsilon \searrow 0} \epsilon^{(\beta+1) / s-2} \sum_{n=1}^{\infty} n^{\beta-2 s+2} \mathbb{E}\left|\tau_{m, n}(f)\right|^{2} I_{\left\{\left|\tau_{m, n}(f)\right| \geq \epsilon n^{s-1}\right\}} \\
=\frac{1}{\beta-2 s+1} \rho^{(\beta+1) / s}[V(f)]^{(\beta+1) / 2 s} \mathbb{E}|\xi|^{(\beta+1) / s},
\end{gathered}
$$

where $\xi \sim N(0,1)$, and

$$
\begin{aligned}
& V(f)=\frac{1}{2 \pi^{2}} \iint_{a(\rho)}^{b(\rho)} f^{\prime}\left(x_{1}\right) f^{\prime}\left(x_{2}\right) \\
& \quad \times \log \left|\frac{\overline{\underline{s}\left(x_{1}\right)}-\underline{s}\left(x_{2}\right)}{\underline{\underline{s}}\left(x_{1}\right)-\underline{s}\left(x_{2}\right)}\right| d x_{1} d x_{2},
\end{aligned}
$$

$\underline{s}(x)=\lim _{z \rightarrow x+i 0} \underline{s}(z)$ and $\underline{s}(z)$ is the Stieltjes transform of $\underline{F}_{2}(y)=(1-y) 1_{[0, \infty)}+y \int_{-\infty}^{y} \gamma_{\rho}(x) d x$, that is, $\underline{s}(z)=$ $\int_{-\infty}^{+\infty}(1 /(x-z)) d \underline{F}_{2}(x)$.

We will give the proofs of theorems in Section 2. Below are a few words about the motivation of this paper. In a sense, our results are similar to the precise asymptotics of independent random variables in the context of complete convergence and moment convergence. Let $X, X_{1}, X_{2}, \ldots$, be i.i.d. random variables and $S_{n}=\sum_{i=1}^{n} X_{i}$; there are a number of results on the convergence of a type of series

$$
\sum_{n=1}^{\infty} \varphi(n) P\left(\left|S_{n}\right| \geq \epsilon f(n)\right), \quad \epsilon>0,
$$

where $\varphi(x)$ and $f(x)$ are the positive functions defined on $[0, \infty)$, and $\sum_{n=1}^{\infty} \varphi(n)=\infty$. In fact, the sum (11) tends to infinity when $\epsilon \searrow 0$, one of the interesting problems is to examine the precise rate at which this occurs, and this amounts to finding a suitable normalizing rate function $\psi(\epsilon)$ such that the sum (11) multiplied by $\psi(\epsilon)$ has a nontrivial limit; this kind of results is frequently called "precise asymptotics." The first result in this direction due to Heyde [3], who proved that $\lim _{\epsilon \downarrow 0} \epsilon^{2} \sum_{n=1}^{\infty} P\left(\left|S_{n}\right| \geq \epsilon n\right)=\mathbb{E} X^{2}$ under the assumptions that $\mathbb{E} X=0$ and $\mathbb{E} X^{2}<\infty$. Some analogous results in more general case can be found in Gut and Spǎtaru [4, 5] and Gut and Steinebach [6]. Moreover, Chow [7] studied the convergence properties of the series 
$\sum_{n=1}^{\infty} n^{p \alpha-2-\alpha} \mathbb{E}\left\{\max _{1 \leq j \leq n}\left|S_{j}\right|-\epsilon n^{\alpha}\right\}_{+}(\epsilon>0)$ when $p \geq 1$, $\alpha>1 / 2, p \alpha>1$. There is a remarkable result obtained by Liu and Lin [8], who considered the precise asymptotics on the second-order moment convergence, which states that

$$
\lim _{\epsilon \unlhd 0} \frac{1}{-\log \epsilon} \sum_{n=1}^{\infty} n^{-2} \mathbb{E} S_{n}^{2} I_{\left\{\left|S_{n}\right| \geq \epsilon n\right\}}=2 \sigma^{2}
$$

when $\mathbb{E} X=0, \mathbb{E} X^{2}=\sigma^{2}$, and $\mathbb{E} X^{2} \log ^{+}|X|<\infty$. Chen and Zhang [9] got the similar results on the second-order moment convergence of empirical process. Furthermore, there are also some precise asymptotic results in other contexts, such as the self-normalized sums, martingale-difference, random fields, and renewal process. It should be mentioned that the corresponding results on random matrices and random growth model have been studied by Su [10], who presented the precise asymptotics of the largest eigenvalues of Gaussian unitary ensembles, Laguerre unitary ensembles, and the longest increasing subsequence of a random permutation. In this paper, we will study the precise asymptotics on the spectral statistics of Wigner matrices and sample covariance matrices, which is also an interesting topic in random matrix theory.

In the rest of the paper, we will always write $N(\epsilon, M)=$ $\left[M \epsilon^{-1 / s}\right]$, where $\epsilon>0$ and $M$ is an arbitrary positive real number. $\|f\|=\sup _{x \in R}|f(x)|$, and $\xi$ stands for a random variable observing the standard Gaussian distribution. We also denote $C$ to be the absolutely positive constant whose value can be different from one place to another.

\section{The Proofs}

Before the main proof of Theorem 3, we will first give four propositions.

Proposition 5. Under the assumptions of Theorem 3, one has

$$
\begin{gathered}
\lim _{\epsilon \searrow 0} \epsilon^{(\beta+1) / s} \sum_{n=1}^{\infty} n^{\beta} P\left(|\xi| \geq \frac{\epsilon n^{s}}{\sqrt{D(f)}}\right) \\
=\frac{1}{\beta+1}[D(f)]^{(\beta+1) / 2 s} E|\xi|^{(\beta+1) / s} .
\end{gathered}
$$

Proof. We calculate that

$$
\begin{aligned}
\lim _{\epsilon \searrow 0} \epsilon^{(\beta+1) / s} \sum_{n=1}^{\infty} n^{\beta} P\left(|\xi| \geq \frac{\epsilon n^{s}}{\sqrt{D(f)}}\right) \\
=\lim _{\epsilon \searrow 0} \epsilon^{(\beta+1) / s} \int_{1}^{\infty} y^{\beta} P\left(|\xi| \geq \frac{\epsilon y^{s}}{\sqrt{D(f)}}\right) d y \\
=\frac{1}{s}[D(f)]^{(\beta+1) / 2 s} \int_{0}^{\infty} t^{(\beta+1) / s-1} P(|\xi| \geq t) d t \\
=\frac{1}{\beta+1}[D(f)]^{(\beta+1) / 2 s} E|\xi|^{(\beta+1) / s} .
\end{aligned}
$$

Proposition 6. Under the assumptions of Theorem 3, one has

$$
\begin{aligned}
& \lim _{\epsilon \searrow 0} \epsilon^{(\beta+1) / s} \sum_{n=1}^{\infty} n^{\beta}\left|P\left(\left|n \eta_{n}(f)\right| \geq \epsilon n^{s}\right)-P\left(|\xi| \geq \frac{\epsilon n^{s}}{\sqrt{D(f)}}\right)\right| \\
& =0 .
\end{aligned}
$$

Proof. We can write

$$
\begin{aligned}
& \sum_{n=1}^{\infty} n^{\beta}\left|P\left(\left|n \eta_{n}(f)\right| \geq \epsilon n^{s}\right)-P\left(|\xi| \geq \frac{\epsilon n^{s}}{\sqrt{D(f)}}\right)\right| \\
& \leq \sum_{n \leq N(\epsilon, M)} n^{\beta}\left|P\left(\left|n \eta_{n}(f)\right| \geq \epsilon n^{s}\right)-P\left(|\xi| \geq \frac{\epsilon n^{s}}{\sqrt{D(f)}}\right)\right| \\
& \quad+\sum_{n>N(\epsilon, M)} n^{\beta} P\left(\left|n \eta_{n}(f)\right| \geq \epsilon n^{s}\right) \\
& \quad+\sum_{n>N(\epsilon, M)} n^{\beta} P\left(|\xi| \geq \frac{\epsilon n^{s}}{\sqrt{D(f)}}\right) \\
& :=I_{1}+I_{2}+I_{3} .
\end{aligned}
$$

Under the assumptions of Theorem 3, the law of each entry $X_{i j}$ also satisify LSI. By Lemma 5 of Vershynin [11], for each fixed positive integer $k, \mathbb{E}\left|X_{i j}\right|^{k}<+\infty$. According to Lemma 1, we can see that

$$
\frac{n \eta_{n}(f)}{\sqrt{D(f)}} \stackrel{d}{\longrightarrow} N(0,1) .
$$

Thus, as $n \rightarrow \infty$,

$$
\Delta_{n}=: \sup _{x \in \mathbb{R}}\left|P\left(\left|n \eta_{n}(f)\right| \geq x\right)-P\left(|\xi| \geq \frac{x}{\sqrt{D(f)}}\right)\right| \longrightarrow 0 .
$$

Using Toeplitz's lemma, we can deduce that

$$
\lim _{\epsilon \searrow 0} \epsilon^{(\beta+1) / s} I_{1}=0 .
$$

For the term $I_{2}$, if $f \in C^{4}(\Pi)$ has a bounded first-order derivative, then $f$ is a Lipschitz function.

By Theorem 8.2 of Bai and Silverstein [12], there exists a real number $C_{1}>0$, such that

$$
\begin{aligned}
\delta_{n}(f) & =\left|\mathbb{E} \int f(x) d F^{M_{n}}(x)-\int f(x) \mu_{s c}(x) d x\right| \\
& \leq C_{1}\left\|f^{\prime}\right\| n^{-1 / 2} .
\end{aligned}
$$


By Lemma 2, for any $\delta_{0}>n \delta_{n}(f)$, there exists $c>0$ such that

$$
P\left(\left|\frac{n \eta_{n}(f)}{\sqrt{D(f)}}\right| \geq \delta_{0}\right) \leq 2 e^{-c\left(\delta_{0}-n \delta_{n}(f)\right)^{2}}
$$

For each fixed $\epsilon>0$, the assumption $s>1 / 2$ reveals that, when $n>N(\epsilon, M)$ with a sufficient large number $M$, we have $\epsilon n^{s}>n \delta_{n}(f)$. Thus there exists $c_{1}>0$, such that

$$
\lim _{M \rightarrow \infty} I_{2} \leq \lim _{M \rightarrow \infty} C \int_{N(\epsilon, M)}^{\infty} x^{\beta} e^{-c_{1} x^{2 s}} d x=0 .
$$

For the term $I_{3}$, as the fact that $\xi \sim N(0,1)$, the wellknown tail probability estimation states that for any $x \in \mathbb{R}$,

$$
P(\xi \geq x) \leq \frac{1}{\sqrt{2 \pi} x} e^{-x^{2} / 2} .
$$

Hence, for any fixed $\epsilon>0$, we can get

$$
\lim _{M \rightarrow \infty} I_{3} \leq \lim _{M \rightarrow \infty} C \int_{N(\epsilon, M)}^{\infty} x^{\beta-1} e^{-x^{2} / 2} d x=0 .
$$

By letting $\epsilon \searrow 0$ and then $M \rightarrow \infty$, a combination of (22) and (24) can get that $\lim _{\epsilon \succ 0} \epsilon^{(\beta+1) / s}\left(I_{2}+I_{3}\right)=0$. Then combined with the relations (16) and (19), we can complete the proof.

Proposition 7. Under the assumptions of Theorem 3, one has

$$
\begin{gathered}
\lim _{\epsilon \searrow 0} \epsilon^{(\beta+1) / s-2} \sum_{n=1}^{\infty} n^{\beta-2 s} \int_{\epsilon n^{s}}^{\infty} y P\left(|\xi| \geq \frac{y}{\sqrt{D(f)}}\right) d y \\
=\frac{s}{(\beta+1)(\beta-2 s+1)}[D(f)]^{(\beta+1) / 2 s} \mathbb{E}|\xi|^{(\beta+1) / s} .
\end{gathered}
$$

Proof. By the similar argument in the proof of Proposition 5, we can show that

$$
\begin{aligned}
& \lim _{\epsilon \searrow 0} \epsilon^{(\beta+1) / s-2} \sum_{n=1}^{\infty} n^{\beta-2 s} \int_{\epsilon n^{s}}^{\infty} y P\left(|\xi| \geq \frac{y}{\sqrt{D(f)}}\right) d y \\
& =\lim _{\epsilon \searrow 0} \epsilon^{(\beta+1) / s-2} D(f) \int_{1}^{\infty} x^{\beta-2 s} \int_{\epsilon x^{s} / \sqrt{D(f)}}^{\infty} y P(|\xi| \geq y) d y \\
& =\lim _{\epsilon \searrow 0} \frac{2}{s}[D(f)]^{1 / 2 s} \int_{\epsilon / \sqrt{D(f)}}^{\infty} t^{1 / s-3} \int_{t}^{\infty} y P(|\xi| \geq y) d y \\
& =\frac{2}{s}[D(f)]^{(\beta+1) / 2 s} \int_{0}^{\infty} y P(|\xi| \geq y) \int_{0}^{y} t^{(\beta+1) / s-3} d t d y \\
& =\frac{s}{(\beta+1)(\beta-2 s+1)}[D(f)]^{(\beta+1) / 2 s} \mathbb{E}|\xi|^{(\beta+1) / s} .
\end{aligned}
$$

Proposition 8. Under the assumptions of Theorem 3, one has

$$
\begin{aligned}
\lim _{\epsilon \searrow 0} \epsilon^{(\beta+1) / s-2} \sum_{n=1}^{\infty} n^{\beta-2 s} & \int_{\epsilon n^{s}}^{\infty} y P\left(\left|n \eta_{n}(f)\right| \geq y\right) d y \\
& -\int_{\epsilon n^{s}}^{\infty} y P\left(|\xi| \geq \frac{y}{\sqrt{D(f)}}\right) d y \mid=0 .
\end{aligned}
$$

Proof. Similar to the argument in the proof of Proposition 6, we can write

$$
\begin{aligned}
& \sum_{n=1}^{\infty} n^{\beta-2 s} \mid \int_{\epsilon n^{s}}^{\infty} y P\left(\left|n \eta_{n}(f)\right| \geq y\right) d y \\
& -\int_{\epsilon n^{s}}^{\infty} y P\left(|\xi| \geq \frac{y}{\sqrt{D(f)}}\right) d y \mid \\
& =\sum_{n=1}^{N(\epsilon, M)} n^{\beta-2 s} \mid \int_{\epsilon n^{s}}^{\infty} y P\left(\left|n \eta_{n}(f)\right| \geq y\right) d y \\
& -\int_{\epsilon n^{s}}^{\infty} y P\left(|\xi| \geq \frac{y}{\sqrt{D(f)}}\right) d y \mid \\
& +\sum_{n>N(\epsilon, M)} n^{\beta-2 s} \mid \int_{\epsilon n^{s}}^{\infty} y P\left(\left|n \eta_{n}(f)\right| \geq y\right) d y \\
& -\int_{\epsilon n^{s}}^{\infty} y P\left(|\xi| \geq \frac{y}{\sqrt{D(f)}}\right) d y \mid \\
& :=J_{1}+J_{2} \text {. }
\end{aligned}
$$

For the term $J_{1}$, we have

$$
\begin{aligned}
J_{1} \leq & \sum_{n=1}^{N(\epsilon, M)} n^{\beta-2 s} \\
& \times \int_{\epsilon n^{s}}^{\infty} y\left|P\left(\left|n \eta_{n}(f)\right| \geq y\right)-P\left(|\xi| \geq \frac{y}{\sqrt{D(f)}}\right)\right| d y \\
\leq & \sum_{n=1}^{N(\epsilon, M)} n^{\beta} \int_{0}^{n^{-s} \Delta_{n}^{-1 / 4}}(x+\epsilon) \\
& \times\left|P\left(\left|n \eta_{n}(f)\right| \geq(x+\epsilon) n^{s}\right)-P\left(|\xi| \geq \frac{(x+\epsilon) n^{s}}{\sqrt{D(f)}}\right)\right| d x \\
& +\sum_{n=1}^{N(\epsilon, M)} n^{\beta}
\end{aligned}
$$




$$
\begin{aligned}
& \quad \times \int_{n^{-s} \Delta_{n}^{-1 / 4}}^{\infty}(x+\epsilon) P\left(\left|n \eta_{n}(f)\right| \geq(x+\epsilon) n^{s}\right) d x \\
& +\sum_{n=1}^{N(\epsilon, M)} n^{\beta} \\
& \quad \times \int_{n^{-s} \Delta_{n}^{-1 / 4}}^{\infty}(x+\epsilon) P\left(|\xi| \geq \frac{(x+\epsilon) n^{s}}{\sqrt{D(f)}}\right) d x \\
& :=\sum_{n=1}^{N(\epsilon, M)} n^{\beta}\left(J_{11}+J_{12}+J_{13}\right) .
\end{aligned}
$$

As $N(\epsilon, M)=\left[M \epsilon^{-1 / s}\right]$, for $n \leq N(\epsilon, M)$, we have $\epsilon \leq M^{s} n^{-s}$. Thus

$$
\begin{aligned}
n^{2 s} J_{11} & \leq n^{2 s} \int_{0}^{n^{-s} \Delta_{n}^{-1 / 4}}(x+\epsilon) \Delta_{n} d x \\
& \leq C n^{2 s} \Delta_{n}\left(n^{-s} \Delta_{n}^{-1 / 4}+M^{s} n^{-s}\right)^{2} \\
& =C\left(\Delta_{n}^{1 / 4}+M^{s} \Delta_{n}^{1 / 2}\right)^{2} \longrightarrow 0 \quad \text { as } n \longrightarrow \infty
\end{aligned}
$$

Noticing that when $\epsilon>0$ is fixed, $\epsilon n^{s}>n \delta_{n}(f)$ for sufficient large $n$, the relation (21) tells us that there exists $c_{1}>0$, such that

$$
\begin{aligned}
& \lim _{n \rightarrow \infty} n^{2 s} J_{12} \\
& =\lim _{n \rightarrow \infty} n^{2 s} \int_{n^{-s} \Delta_{n}^{-1 / 4}}^{\infty}(x+\epsilon) P\left(\left|n \eta_{n}(f)\right| \geq(x+\epsilon) n^{s}\right) d x \\
& =\lim _{n \rightarrow \infty} \int_{\epsilon n^{s}+\Delta_{n}^{-1 / 4}}^{\infty} y P\left(\left|n \eta_{n}(f)\right| \geq y\right) d y \\
& \leq \lim _{n \rightarrow \infty} C \int_{\epsilon n^{s}+\Delta_{n}^{-1 / 4}}^{\infty} y e^{-c_{1} y^{2}} d y \\
& \leq \lim _{n \rightarrow \infty} C \int_{\Delta_{n}^{-1 / 4}}^{\infty} y e^{-c_{1} y^{2}} d y \\
& \leq \lim _{n \rightarrow \infty} C e^{-c_{1} \Delta_{n}^{-1 / 2}}=0 .
\end{aligned}
$$

By the same argument as for $J_{12}$, using the relation (23), we can easily prove that

$$
\lim _{n \rightarrow \infty} n^{2 s} J_{13}=0 .
$$

Hence, we have proved that $\lim _{n \rightarrow \infty} \Xi_{n}(\epsilon)$ := $\lim _{n \rightarrow \infty} n^{2 s}\left(J_{11}+J_{12}+J_{13}\right)=0$ uniformly for $\epsilon>0$. By Toeplitz's lemma again, we can further deduce that

$$
\begin{aligned}
& \lim _{\epsilon \searrow 0} \epsilon^{(\beta+1) / s-2} J_{1} \\
& =\lim _{\epsilon \searrow 0} \epsilon^{(\beta+1) / s-2} \sum_{n=1}^{N(\epsilon, M)} n^{\beta}\left(J_{11}+J_{12}+J_{13}\right) \\
& \leq \lim _{\epsilon \searrow 0} C[N(\epsilon, M)]^{2 s-\beta-1} \sum_{n=1}^{N(\epsilon, M)} n^{\beta-2 s} \Xi_{n}(\epsilon)=0 .
\end{aligned}
$$

For the term $J_{2}$, we can write

$$
\begin{aligned}
J_{2} \leq & \sum_{n>N(\epsilon, M)} n^{\beta-2 s} \\
& \quad \times \int_{\epsilon n^{s}}^{\infty} y\left[P\left(\left|n \eta_{n}(f)\right| \geq y\right)+P\left(|\xi| \geq \frac{y}{\sqrt{D(f)}}\right)\right] d y \\
:= & J_{21}+J_{22} .
\end{aligned}
$$

By using the relation (21) again, for any fixed $\epsilon>0$, when $M \rightarrow \infty$, there exists $c_{1}>0$, such that

$$
\begin{aligned}
& \epsilon^{(\beta+1) / s-2} J_{21} \\
& \leq C \epsilon^{(\beta+1) / s-2} \sum_{n>N(\epsilon, M)} n^{\beta-2 s} \int_{n}^{\infty} \epsilon^{2} x^{2 s-1} P\left(\left|n \eta_{n}(f)\right| \geq \epsilon x^{s}\right) d x \\
& \leq C \epsilon^{(\beta+1) / s} \sum_{n>N(\epsilon, M)} n^{\beta-2 s} \int_{n}^{\infty} x^{2 s-1} e^{-c_{1} \epsilon^{2} x^{2 s}} d x \\
& \leq C \epsilon^{(\beta+1) / s} \int_{N(\epsilon, M)}^{\infty} y^{\beta-2 s} \int_{y}^{\infty} x^{2 s-1} e^{-c_{1} \epsilon^{2} x^{2 s}} d x d y \\
& \leq C \epsilon^{(\beta+1) / s} \int_{N(\epsilon, M)}^{\infty} x^{\beta-2 s+1} x^{2 s-1} e^{-c_{1} \epsilon^{2} x^{2 s}} d x \\
& \leq C \int_{M^{s}}^{\infty} y^{(\beta+1) / s-1} e^{-c_{1} y^{2}} d y \longrightarrow 0 .
\end{aligned}
$$

Hence, by letting $\epsilon \searrow 0$ firstly and then taking $M \rightarrow \infty$, we get $\lim _{\epsilon \backslash 0} \epsilon^{(\beta+1) / s-2} J_{21}=0$.

Following the proof of $J_{21}$ and using the relation (23), we can easily prove that $\epsilon^{(\beta+1) / s-2} J_{22}$ vanishes as $\epsilon \searrow 0$, which yields that

$$
\lim _{\epsilon \searrow 0} \epsilon^{(\beta+1) / s-2} J_{2}=0 .
$$

By combining (33) and (36), we conclude that the proof is completed.

Proof of Theorem 3. According to the fact that for any random variable $\zeta$ and $a \in \mathbb{R}$,

$$
\mathbb{E} \zeta I_{\{\zeta \geq a\}}=a P(\zeta \geq a)+\int_{a}^{\infty} P(\zeta \geq t) d t,
$$

we can see that

$$
\begin{aligned}
\mathbb{E}\left|\eta_{n}(f)\right|^{2} I_{\left\{\left|\eta_{n}(f)\right| \geq \epsilon n^{s-1}\right\}} & =\epsilon^{2} n^{2 s-2} P\left(\left|\eta_{n}(f)\right| \geq \epsilon n^{s-1}\right) \\
& +2 n^{-2} \int_{\epsilon n^{s}}^{\infty} y P\left(\left|n \eta_{n}(f)\right| \geq y\right) d y .
\end{aligned}
$$

By Propositions 5-8, we can get Theorem 3 easily. 
Proof of Theorem 4. The proof is essentially the same as for Theorem 3, which mainly depends on the central limit theorem of the spectral statistics and the concentration inequality of the empirical spectral measure. We will only list some key tools in the proof, and the details are omitted here.

Lemma 9 (see [13]). Consider the sample covariance matrix $W_{m, n}=(1 / n) Y_{m n} Y_{m n}^{*}$ as above, where $E\left|Y_{i j}\right|^{4}=2$ and $E\left|Y_{i j}\right|^{8}<$ $\infty$ for all $1 \leq i \leq m, 1 \leq j \leq n, m=[n \rho](0<\rho \leq 1)$. Let

$$
\widetilde{G}_{m, n}(f)=m\left\{\int f(x) d F^{W_{m, n}}(x)-\int f(x) \gamma_{m / n}(x) d x\right\}
$$

and denote $\Gamma$ to be an open interval including $[a(\rho), b(\rho)]$. Then the empirical processes $\left\{\widetilde{G}_{m, n}(f): f \in C^{4}(\Gamma)\right\}$ converge weakly in finite dimension to a Gaussian process $\left\{\widetilde{G}(f): f \in C^{4}(\Gamma)\right\}$ with mean zero and the covariance function $\overline{\mathrm{Cov}}(f, g)$ given by

$$
\begin{aligned}
& \widetilde{\operatorname{Cov}}(f, g)=\frac{1}{2 \pi^{2}} \iint_{a(\rho)}^{b(\rho)} f^{\prime}\left(x_{1}\right) g^{\prime}\left(x_{2}\right)
\end{aligned}
$$

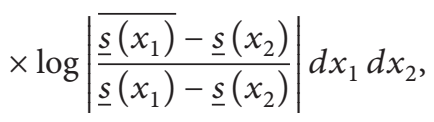

$$
\begin{aligned}
& f, g \in C^{4}(\Gamma),
\end{aligned}
$$

where $\underline{s}(x)$ is defined in Theorem 4.

Lemma 10. Assume $f$ is a Lipschitz function and $\tau_{m, n}(f)$ is defined by (8). Denote $\widetilde{\delta}_{m, n}(f)=\mid \mathbb{E} \int f(x) d F^{W_{m, n}}(x)-$ $\int f(x) \gamma_{m / n}(x) d x \mid$. Then under the assumptions of Theorem 4, for any $\delta>\widetilde{\delta}_{m, n}(f)$, there exists $c_{2}>0$, such that

$$
P\left(\left|\tau_{m, n}(f)\right| \geq \delta\right) \leq 2 e^{-c_{2} m^{2}\left(\delta-\widetilde{\delta}_{m, n}(f)\right)^{2}} .
$$

Proof. If we denote

$$
\tau_{m, n}^{\circ}(f)=\int f(x) d F^{W_{m, n}}(x)-E\left[\int f(x) d F^{W_{m, n}}(x)\right],
$$

then by the Corollary 1.8(b) in Guionnet and Zeitouni [2], for any $\delta>0$, there exists $c_{2}>0$ such that

$$
P\left(\left|\tau_{m, n}^{\circ}(f)\right| \geq \delta\right) \leq 2 e^{-c_{2} \delta^{2} m^{2}} .
$$

As we can write

$$
\begin{aligned}
& P\left(\left|\tau_{m, n}(f)\right| \geq \delta\right) \\
& \leq P\left(\left|\tau_{m, n}^{\circ}(f)\right|\right. \\
& \left.\quad+\left|\mathbb{E} \int f(x) d F^{W_{m, n}}(x)-\int f(x) \gamma_{m / n}(x) d x\right| \geq \delta\right) \\
& \leq P\left(\left|\tau_{m, n}^{\circ}(f)\right| \geq \delta-\widetilde{\delta}_{m, n}(f)\right),
\end{aligned}
$$

for each $\delta>\widetilde{\delta}_{m, n}(f)$, we have

$$
P\left(\left|\tau_{m, n}(f)\right| \geq \delta\right) \leq 2 e^{-c_{2} m^{2}\left(\delta-\widetilde{\delta}_{m, n}(f)\right)^{2}}
$$

Just like the proof of Proposition 6, in order to use Lemma 10, we will need to estimate the order of $\widetilde{\delta}_{m, n}(f)$, and the following remark is needed.

Remark 11. For the estimation of $\widetilde{\delta}_{m, n}(f)$, by the result of Bai et al. [14], we can see that

$$
\sup _{x \in \mathbb{R}}\left|\mathbb{E} F_{2}^{(m, n)}(x)-F_{2}^{(m, n)}(x)\right| \leq C m^{-1 / 2},
$$

where $F_{2}^{(m, n)}(x)=\int_{0}^{x} d F^{W_{m, n}}(t)$ is the empirical spectral distribution of $W_{m, n}$. As a direct consequence, when $f$ is differentiable and $f^{\prime}$ is bounded, there exists a real number $C_{2}>0$, such that $\tilde{\delta}_{m, n}(f) \leq C_{2}\left\|f^{\prime}\right\| m^{-1 / 2}$.

\section{Acknowledgments}

This work is supported by the National Natural Science Foundation of China (Grant nos. 11326173 and 11301146) and the Foundation of Henan Educational Committee in China (Grant no. 13A110087).

\section{References}

[1] Z. D. Bai, X. Y. Wang, and W. Zhou, "CLT for linear spectral statistics of Wigner matrices," Electronic Journal of Probability, vol. 14, article 83, pp. 2391-2417, 2009.

[2] A. Guionnet and O. Zeitouni, "Concentration of the spectral measure for large matrices," Electronic Communications in Probability, vol. 5, pp. 119-136, 2000.

[3] C. C. Heyde, "A supplement to the strong law of large numbers," Journal of Applied Probability, vol. 12, pp. 173-175, 1975.

[4] A. Gut and A. Spătaru, "Precise asymptotics in the BaumKatz and Davis laws of large numbers," Journal of Mathematical Analysis and Applications, vol. 248, no. 1, pp. 233-246, 2000.

[5] A. Gut and A. Spătaru, "Precise asymptotics in the law of the iterated logarithm," The Annals of Probability, vol. 28, no. 4, pp. 1870-1883, 2000.

[6] A. Gut and J. Steinebach, "Precise asymptotics-a general approach," Acta Mathematica Hungarica, vol. 138, no. 4, pp. 365-385, 2013.

[7] Y.S. Chow, "On the rate of moment convergence of sample sums and extremes," Bulletin of the Institute of Mathematics, vol. 16, no. 3, pp. 177-201, 1988.

[8] W. D. Liu and Z. Y. Lin, "Precise asymptotics for a new kind of complete moment convergence," Statistics \& Probability Letters, vol. 76, no. 16, pp. 1787-1799, 2006.

[9] Y.-Y. Chen and L.-X. Zhang, "Second moment convergence rates for uniform empirical processes," Journal of Inequalities and Applications, vol. 2010, Article ID 972324, 9 pages, 2010.

[10] Z. G. Su, "Precise asymptotics for random matrices and random growth models," Acta Mathematica Sinica, vol. 24, no. 6, pp. 971$982,2008$. 
[11] R. Vershynin, "Introduction to the non-asymptotic analysis of random matrices," in Compressed Sensing, Y. Eldar and G. Kutyniok, Eds., pp. 210-268, Cambridge University Press, Cambridge, UK, 2012.

[12] Z. D. Bai and J. W. Silverstein, Spectral Analysis of Large Dimensional Random Matrices, Science Press, Beijing, China, 2006.

[13] Z. D. Bai, X. Y. Wang, and W. Zhou, "Functional CLT for sample covariance matrices," Bernoulli, vol. 16, no. 4, pp. 1086-1113, 2010.

[14] Z. D. Bai, B. Q. Miao, and J.-F. Yao, "Convergence rates of spectral distributions of large sample covariance matrices," SIAM Journal on Matrix Analysis and Applications, vol. 25, no. 1, pp. 105-127, 2003. 


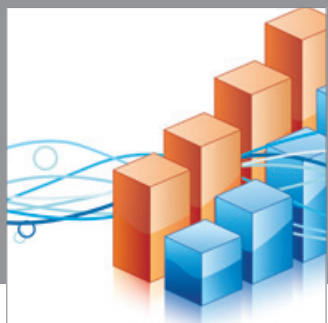

Advances in

Operations Research

mansans

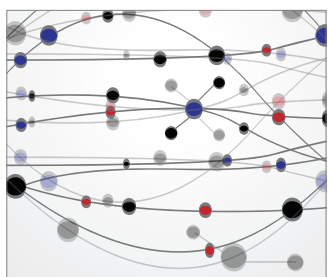

The Scientific World Journal
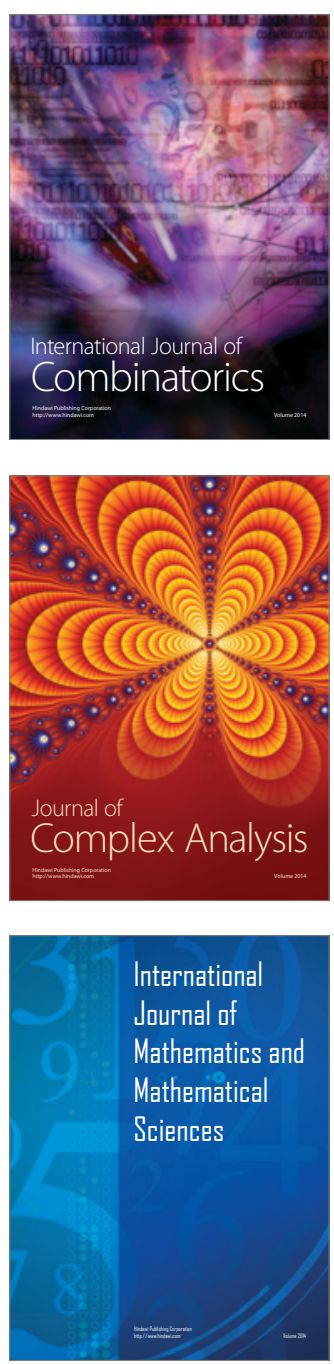
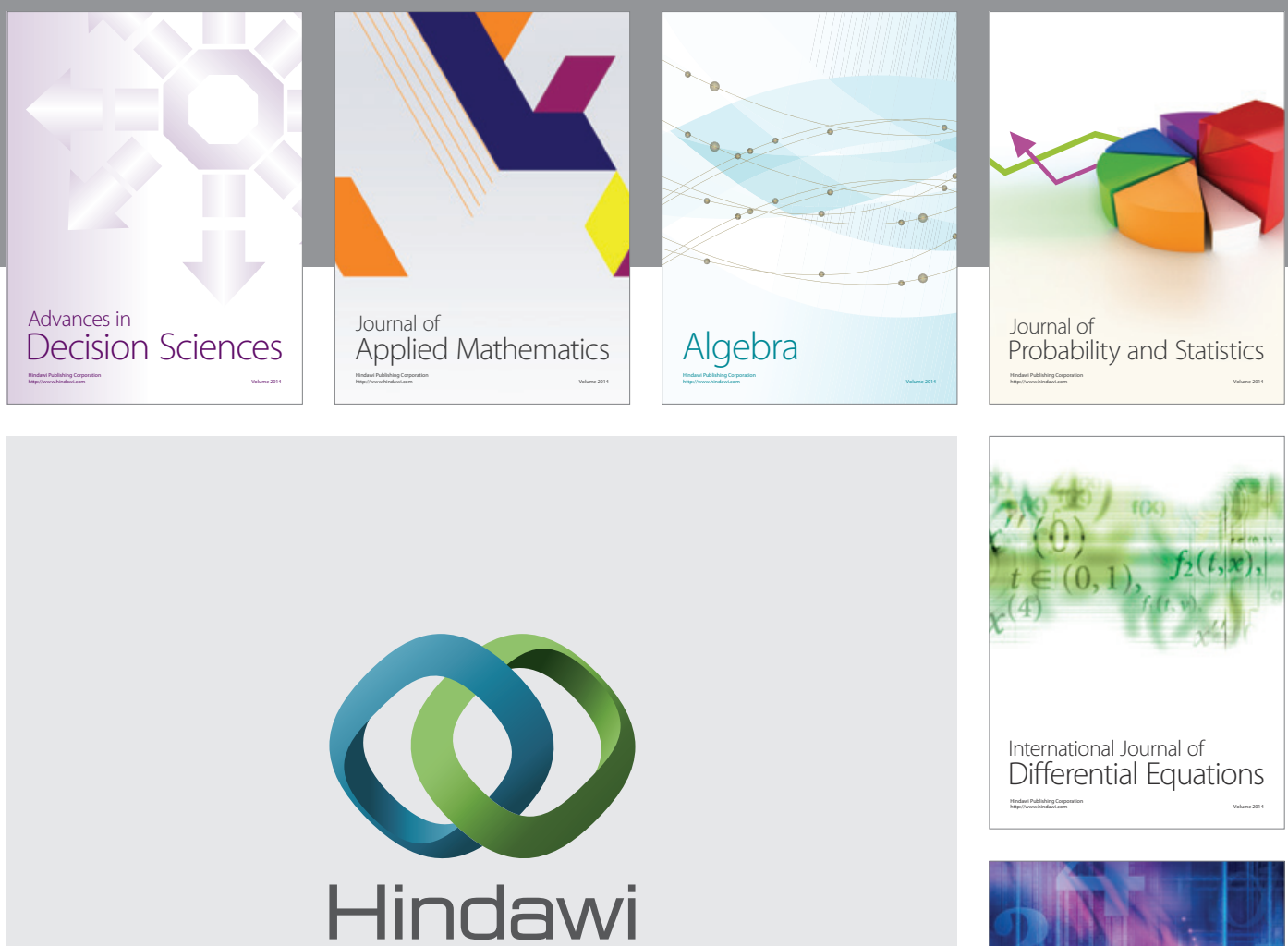

Submit your manuscripts at http://www.hindawi.com
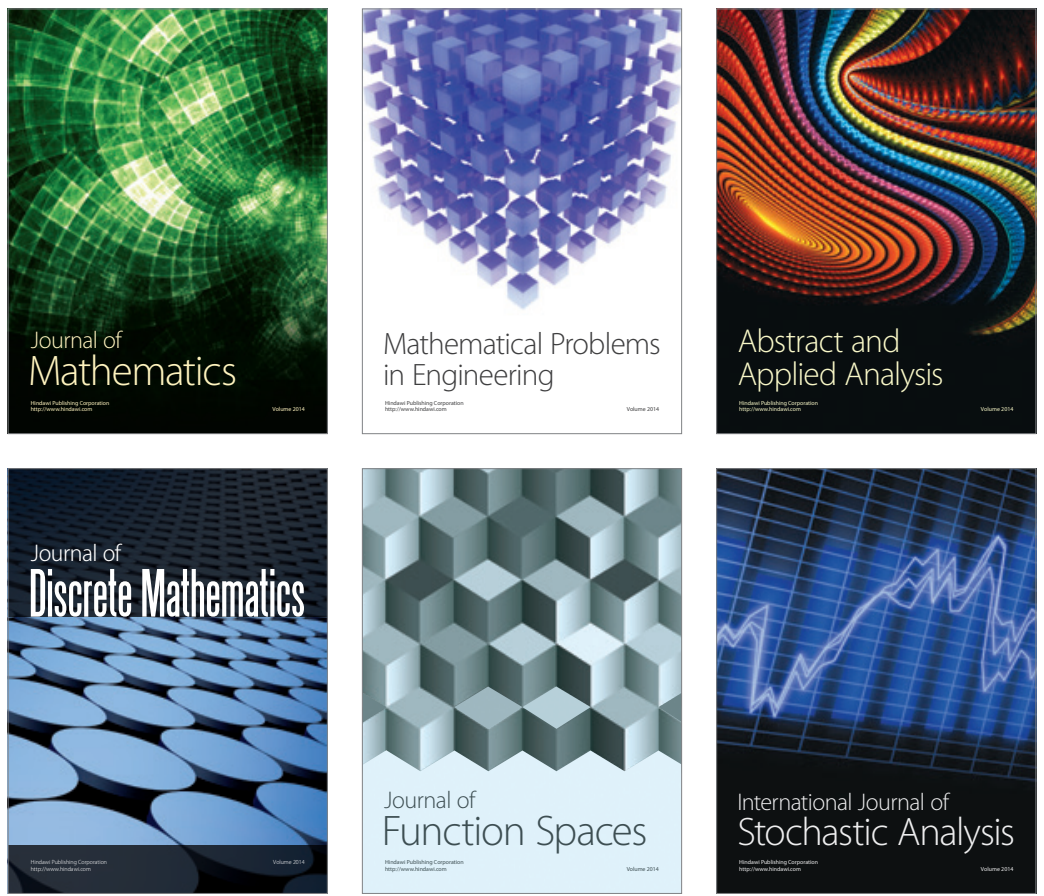

Journal of

Function Spaces

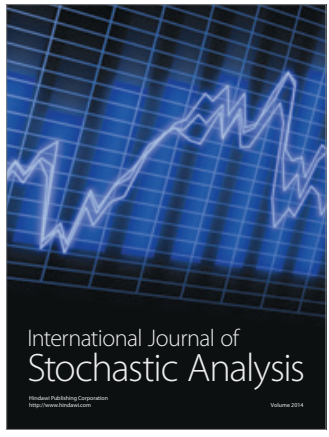

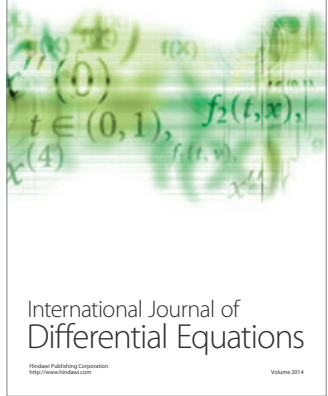
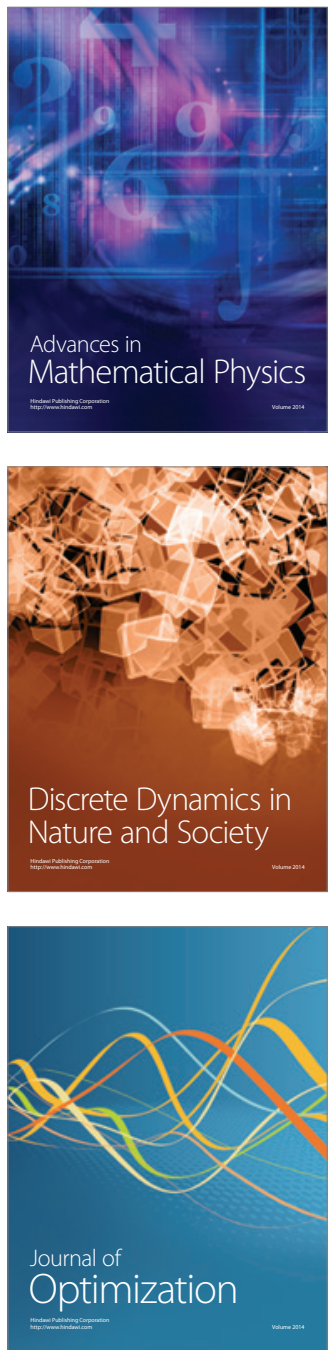